Les ANNALES Les Annales de droit

DE DROIT

$12 \mid 2018$

Varia

\title{
L'avènement de la coproduction de la sécurité : le bilan et les acteurs
}

The emergence of the co-production of security: an overview and actors

\section{Héritage Bita Heyeghe}

\section{(2) OpenEdition}

Journals

Édition électronique

URL : http://journals.openedition.org/add/978

DOI : $10.4000 /$ add. 978

ISSN : 2606-1988

Éditeur

Presses universitaires de Rouen et du Havre

Édition imprimée

Pagination : 61-85

ISBN : 979-10-240-0937-7

ISSN : 1955-0855

Référence électronique

Héritage Bita Heyeghe, "L'avènement de la coproduction de la sécurité : le bilan et les acteurs 》, Les

Annales de droit [En ligne], 12 | 2018, mis en ligne le 05 février 2019, consulté le 05 décembre 2019.

URL : http://journals.openedition.org/add/978; DOI : 10.4000/add.978

Presses universitaires de Rouen et du Havre 


\title{
L'avènement de la coproduction de la sécurité : le bilan et les acteurs
}

\author{
Héritage Bita Heyeghe
}

Aux termes de l'article 12 de la Déclaration des droits de l'homme et du citoyen de 1789 (DDHC), il est affirmé que «la garantie des droits de l'Homme et du Citoyen nécessite une force publique: cette force est donc instituée pour l'avantage de tous, et non pour l'utilité particulière de ceux auxquels elle est confiée ». C'est dans ce même ordre d'idées que Christian Vigouroux affirme que «la force est l'affaire de la nation ${ }^{1}$ ». Reprenant ces deux assertions, il apparaît que l'usage de la force relève principalement du rôle de l'État qui doit d'une part, garantir l'intégrité de son territoire non seulement contre des formes d'attaques qui s'y produiraient, mais également contre celles qui émaneraient de l'extérieur; et d'autre part, assurer la protection de ses citoyens et en cas de nécessité, les assujettir à des formes de contrainte. Ce rôle est un invariant, en temps de paix comme en temps de crise, l'État «doit disposer de moyens aussi efficaces qu'exceptionnels [pour le remplir ${ }^{2}$ ]».

Par ailleurs, en dehors des considérations tenant à la protection du territoire national et des citoyens, la mise en œuvre de cette force doit contribuer à l'exercice par ces derniers d'un ensemble de droits et libertés consacrés parmi lesquels figurent notamment la sûreté, la sécurité, la liberté d'aller et venir, la liberté de culte, la liberté du commerce et de l'industrie ${ }^{3}$. Dans cette étude, c'est à la sécurité que nous nous consacrerons en nous intéressant à la méthode de coproduction dont elle est désormais empreinte. Pour ce faire, il importe tout d'abord d'éclairer le lecteur sur ces deux termes de «coproduction » et de «sécurité».

1. Christian Vigouroux, Du juste exercice de la force, Paris, Odile Jacob, 2017, p. 272.

2. Jean-Philippe Derosier, "L'état d'urgence : un régime d'exceptionnel et provisoire», JCP A 2015, p. 2-3.

3. Art. 2 de la DDHC; al. 11 du préambule de la Constitution du 27 octobre 1946; art. $1^{\text {er }}$ et 4 de la DDHC; art. 10 de la DDHC et, dans une certaine mesure, al. 5 du préambule de la Constitution de 1946. 
Selon Le Petit Robert, la coproduction renvoie à l'idée d'une "production par plusieurs producteurs» autrement dit, elle renvoie à l'association d'un ensemble de producteurs en vue de la réalisation d'une œuvre déterminée ${ }^{4}$. D’un point de vue sociojuridique, la coproduction désignerait «les nouveaux modes d'actions» de l'État qui se manifestent par le recours à « des formes de partenariats » dans le but de répondre aux besoins de la population (dans notre cas, les besoins de sécurité ${ }^{5}$ ). Ainsi, elle apparaitrait comme une technique contractuelle ${ }^{6}$ qui, au-delà de mobiliser autour d'elle une diversité d'acteurs, favoriserait notamment leur partenariat, la mutualisation de leur expérience, leurs moyens et actions ainsi que la coordination de ces dernières pour lutter contre l'insécurité ressentie par les citoyens ${ }^{7}$. Dans le cas qui nous intéresse, sont concernés par celle-ci : l'État, les collectivités territoriales, les magistrats, les associations, les bailleurs sociaux, etc. En outre, elle souligne que, tant la production de la sécurité que sa gestion ne sauraient désormais relever d'un seul niveau de décision et d'acteurs, d'autant plus que l'insécurité et les actes de violence dont sont victimes les citoyens, sont de plus en plus protéiformes. Daniel Vaillant n'avait-il pas proclamé en 2001 que "la sécurité est l'affaire de tous ${ }^{8}$ "?

S'agissant du terme "sécurité », il peut également être envisagé sous un aspect général, celui présentant la sécurité comme «l'état d'esprit confiant et tranquille d'une personne qui se croit à l'abri du danger ${ }^{9}$ ». À côté de cette conception générale, une conception organique peut être retenue; elle se fonde "sur la nature juridique des personnes chargées des missions de sécurité ». De ce point de vue, elle sera qualifiée

4. Le Petit Robert: dictionnaire alphabétique et analogique de la langue française, Paris, Le Robert, 2011, p. 542. Ce terme est essentiellement utilisé dans le domaine du cinéma et du spectacle pour signaler la production «(d'un film, d'un spectacle) [par des producteurs] souvent de nationalités différentes».

5. Frédéric Diaz, "coproduction" de la sécurité: une nouvelle forme de l'interventionnisme étatique pour une meilleure sécurité du public?", Déviance et société, vol. $27, \mathrm{n}^{\mathrm{o}} 4$, 2003, p. 429-458. Voir également les travaux de Virginie Gautron, «La coproduction locale de la sécurité en France : un partenariat interinstitutionnel déficient», Champ pénal/Penal Field, vol. VII, 2010, http://champpenal.revues.org/ 7719. L'auteur présente également la coproduction comme « une nouvelle doctrine d'action publique qui s'est progressivement imposée » en matière de sécurité.

6. Quand bien même il ne s'agit pas du contrat au sens commun du droit privé ou administratif, la mise en œuvre de la coproduction révèlerait l'existence d'une forme de contrat conclu entre ses acteurs.

7. Voir en ce sens, Sébastian Roché, Le sentiment d’insécurité, Paris, PUF, 1993, 311 p.

8. Daniel Vaillant lors du débat sur la loi ${ }^{0}$ 2001-1062 du 15 novembre 2001 relative à la sécurité quotidienne.

9. Le Petit Robert, op. cit., p. 2339. 
de sécurité publique lorsqu'elle "est assurée par l'État et les autres personnes publiques» ou de sécurité privée si elle «est assurée par des entreprises privées de sécurité ${ }^{10} »$.

S'y ajoutent enfin d'une part, la conception matérielle prenant en compte "l'étendue du monopole de l'État sur les activités de sécurité", elle s'intéresse donc aux tâches pour lesquelles des acteurs sont mobilisés; d'autre part, la conception téléologique « en vertu de laquelle la sécurité [publique poursuivrait] un objectif d'intérêt général» et la conception fonctionnelle qui signale "la manière dont [la sécurité protège] les personnes ». Soit elle protège "les intérêts de personnes individualisées », soit elle protège ceux "d'un groupe de personnes indifférenciées et prises collectivement ${ }^{11} »$. C'est cette conception fonctionnelle que nous proposons d'appliquer au terme "sécurité», c'est-à-dire, au sens de la police; cette "activité administrative dont l'objet est d'assurer l'ordre public $^{12} »$ au profit de la collectivité.

Pour ponctuer cette présentation préliminaire, précisons en outre que la sécurité dont il est ici question est celle qui tend à lutter contre les troubles sociaux, les actes de violence, la délinquance, les attaques terroristes, etc., et à préserver les biens. La présente étude s'intéressera aux politiques publiques de sécurité élaborées pour y faire face.

$\mathrm{Au}$ demeurant, le sujet soumis à notre analyse permet de rendre compte du fait que la production de la sécurité des personnes et des biens est désormais marquée par une approche partenariale, son origine n'est plus uniquement étatique. Cet état de fait mérite attention, non seulement en revenant, une vingtaine d'années après son avènement, sur les mécanismes de matérialisation de la coproduction de la sécurité, les buts poursuivis par elle, mais également sur les résultats qu'on peut y relever et son avenir pour souligner ce que cette politique de sécurité révèle sur l'État en général et, en particulier, sur la gestion de la sécurité. Enfin, la question de l'articulation entre les acteurs de la coproduction aux différents niveaux de l'État sera également abordée.

10. Jean-François Lafaix, «La privatisation des activités de police et de sécurité », communication présentée au cours des $12^{\mathrm{e}}$ journées juridiques franco-allemandes organisées par la Société de législation comparée sur le thème "Sécurité et libertés ", les 11 et 12 novembre 2016 à Postdam (Allemagne).

11. Ibid. Dans le premier cas, la sécurité sera dite privée, tandis que dans le second elle aura un caractère public.

12. Voir Étienne Picard, «Police», dans Denis Alland et Stéphane Rials (dir.), Dictionnaire de la culture juridique, Paris, PUF, 2003, p. 1163. Nous rappelons pour le contenu (matériel) de l'ordre public, l'article L.2212-2 du CGCT. 
Dans cette perspective, notre réflexion portera sur deux idées principales. La première s'intéressera à la construction de la coproduction de la sécurité (1); la seconde mettra en évidence ses acteurs (2).

\section{La construction de la coproduction de la sécurité}

La méthode de coproduction s'est construite autour de son introduction dans les politiques publiques de sécurité (1.1). Elle tend à s'affermir dans le système sécuritaire national, ce qui conduit à en dresser un bilan (1.2).

\subsection{Les politiques publiques de sécurité, fondement de la méthode de coproduction}

Depuis les années 1970, l'État est confronté à la montée en puissance des vols, des actes de violence et de la délinquance urbaine, générant un ressenti d'insécurité voire de peur. De nos jours, c'est à des formes particulières de troubles sociaux qu'il doit s'attaquer (notamment les menaces et les attaques terroristes). Face à de telles situations, il a initié des politiques publiques de prévention et de répression pour répondre à une demande sociale croissante de sécurité ${ }^{13}$. Des travaux préparatoires aboutissant à l'élaboration de rapports ont souvent précédé ces politiques. Nous pouvons présenter deux d'entre eux : le rapport d'Alain Peyrefitte de $1976^{14}$ et celui de Gilbert Bonnemaison de $1982^{15}$.

Ces deux rapports avaient pour objectif de fixer les grandes orientations en matière de prévention, de répression et de lutte contre l'insécurité. Leur célébrité est toujours d'actualité et les études entreprises dans le champ de la sécurité - au sens large du terme - ne manquent pas de s'y référer. Concrètement, l'esprit général de ces travaux était d'introduire

13. Pour une définition de la notion de politique publique et son application en matière de prévention et de répression de la délinquance, voir par exemple, Virginie Gautron, Les politiques publiques de lutte contre la délinquance, thèse de doctorat, Université de Nantes, 2006, p. 17 et suiv., http://hal.archives-ouvertes.fr/ tel-00813174/document. Nous renvoyons également pour des développements plus complets sur les politiques publiques de sécurité (au niveau local), l'ouvrage de Nadine Dantonel-Cor et Gérard Marcou (dir.), Les politiques publiques locales de sécurité intérieure, Paris, L'Harmatthan, 2015, 314 p.

14. Du nom du président du Comité sur la violence, la criminalité et la délinquance, Réponse à la violence: rapport à M. Le Président de la République, Paris, La Documentation française, 1977.

15. Du nom du président de la Commission des maires sur la sécurité, Face à la délinquance: prévention, répression, solidarité, Paris, La Documentation française, 1982, rapport remis au Premier ministre. 
une nouvelle approche en matière de lutte contre l'insécurité. Cette approche se voulait globale, autrement dit, les auteurs de ces rapports invitaient l'État (principalement) à penser les causes des violences et de la délinquance, leurs territoires et leurs auteurs, mais surtout ils militaient pour une intégration plus large des différents acteurs susceptibles de lutter contre.

Cette coproduction de la sécurité souhaitée s'est matérialisée au cours des années 1980 par un partenariat noué essentiellement entre l'État central et les collectivités territoriales. La création, au niveau national, des conseils nationaux de prévention de la délinquance et au niveau local, des conseils communaux de prévention de la délinquance (CCPD) ainsi que des conseils départementaux de prévention de la délinquance (CDPD), est par exemple le fruit de ce partenariat. Par ailleurs, ce partenariat s'est également traduit par la mise en œuvre de la politique dite de «la ville» dont l'objectif était de «recherche[r] des solutions préventives globales, et non sectorielles, aux problèmes sociaux ${ }^{16} »$; les contrats de prévention pour la sécurité dans la ville ont été les dispositifs retenus en la matière.

En outre, il peut être soutenu que son introduction formelle dans les politiques publiques de sécurité a été effective grâce aux dispositions de l'article $1^{\text {er }}$ de la loi d'orientation et de programmation relative à la sécurité ${ }^{17}$ qui affichaient leur volonté d'associer à sa réalisation, non seulement les moyens de l'État, des collectivités territoriales, mais surtout ceux des acteurs privés ${ }^{18}$; ce qui permettrait à ces acteurs « [de] dialoguer sur leurs missions respectives, [de] définir des orientations communes, [de] mutualiser leurs savoir-faire, leurs ressources et [de]

16. «Les politiques locales de sécurité », Vie publique, 2009, http://www.vie-publique.fr/ decouverte-institutions/institutions/approfondissements/politiques-localessecurite.html. Dans ce domaine, c'est la Délégation interministérielle à la ville qui procédait à une sorte de diagnostic et d'évaluation des dispositifs locaux de sécurité notamment des CCPD. Pour d'autres développements sur la politique de la ville, voir par exemple, Jean-Bernard Auby, Droit de la ville: du fonctionnement juridique des villes au droit à la ville, Paris, LexisNexis, 2013.

17. L. $\mathrm{n}^{\mathrm{o}}$ 95-73, 21 janv. 1995, ledit article $1^{\mathrm{er}}$ est désormais abrogé, la loi quant à elle a été modifiée en 2003 par la L. $n^{0}$ 2003-239, 18 mars 2003 pour la sécurité intérieure. L'abrogation de cet article n'a pas porté à conséquence quant à l'affirmation de la dynamique de la coproduction de la sécurité.

18. En ce sens, cette loi semble être l'une des premières dispositions qui ait clairement affiché la reconnaissance de la sécurité privée comme participant à la sécurité des personnes et des biens. Le texte dispose en effet que les entreprises privées de sécurité " concourent à la sécurité générale (Annexe I, rapports sur les orientations de la politique de sécurité; 3 . Les activités privées de sécurité). 
coordonner leurs actions ${ }^{19} »$. Y sont par ailleurs adjointes certaines politiques de territorialisation ${ }^{20}$.

Les périodes clés de la construction de la coproduction de la sécurité ayant été brièvement rappelées, il est intéressant de présenter les quelques dispositifs qui la matérialisent. De ce point de vue, ces dispositifs sont de deux sortes : dispositifs de concertation d'une part et dispositifs dits opérationnels d'autre part.

S'agissant des dispositifs de concertation, nous remarquons tout d'abord qu'ils sont essentiellement d'émanation étatique et qu'ils ont vocation à s'appliquer sur l'ensemble du territoire national et qu'ensuite ils ont une dimension interministérielle et collégiale. Nous pouvons présenter quelques-uns des principaux dispositifs : le Conseil de défense et sécurité nationale, l'ancien Conseil de sécurité intérieure ${ }^{21}$; le Conseil interministériel de prévention de la délinquance $\left(\mathrm{CIPD}^{22}\right)$; le Comité interministériel des villes $\left(\mathrm{CIV}^{23}\right)$; enfin la Mission interministérielle de lutte

19. Virginie Gautron, art. cité.

20. La territorialisation renvoie à une organisation ayant vocation "à produire un meilleur ajustement [des politiques publiques] aux besoins [des populations], [à] une gestion adaptée aux réalités et contraintes locales, ainsi qu'un suivi plus fin des programmes» (Virginie Gautron, art. cité). La territorialisation est «la stratégie qui amène le pouvoir central à déconcentrer son action tout en composant avec les pouvoirs locaux, de manière à instaurer une véritable gouvernance territoriale » (Grégoire Doré, "La territorialisation de la sécurité intérieure : mythe ou réalité ? », dans Frédéric Debove et Olivier Renaudie (dir.), Sécurité intérieure. Les nouveaux défis, Paris, Vuibert, 2013, p. 96).

21. Depuis 2010, ce conseil s'est substitué au Conseil de sécurité intérieure créé en 2002 par Jacques Chirac, alors Premier ministre, après les attentats survenus à l'automne 1986. Ses principales missions sont la définition des orientations en matière de politique militaire, de dissuasion, de conduite des opérations militaires extérieures, de lutte contre le terrorisme. Il intervient également en matière de sécurité intérieure et, à ce titre, il prend en charge la coordination et le développement de la lutte contre la délinquance.

22. Il est ainsi désigné depuis le décret $\mathrm{n}^{\mathrm{0}}$ 2016-553 du 6 mai 2016, portant modification des dispositions relatives à la prévention de la délinquance.

23. Relevant du ministère de la Cohésion des territoires ayant en charge la politique de la ville, le CIV est l'instance de décision de la politique de la ville. Il est composé du Premier ministre ou du ministre en charge de la politique de la ville, ainsi que de tous les ministères concernés par cette politique. Il veille à la prise en compte par les ministères concernés, notamment, de la spécificité des quartiers sensibles dans leurs politiques et leurs moyens budgétaires, http://www.ville.gouv.fr/?le-comiteinterministeriel-des, 388 . 
contre la drogue et la toxicomanie, actuelle Mission interministérielle de lutte contre les drogues et les conduites addictives ${ }^{24}$.

C'est au sein de ces dispositifs qu'est définie la politique de coproduction de la sécurité, que les acteurs de la sécurité des personnes et des biens dialoguent entre eux, unifient leurs modes de fonctionnement et coordonnent leurs interventions. Ces dispositifs révèlent une certaine fracture des dichotomies (droit privé, droit public, État, collectivités territoriales, police judiciaire, police administrative, etc.) enfermant les acteurs dans leurs champs d'intervention traditionnels; ils favorisent une gestion plus globale et intégrative de la sécurité, les actes de violences et de délinquance devenant protéiformes, si bien que les domaines « du pénal » et « du social » sont eux-mêmes marqués par une «interpénétration progressive $^{25}$ ", ce qui implique une mutation des pratiques (recours aux échanges d'informations, à la complémentarité, etc.).

La lutte contre le terrorisme offre également une illustration de la coproduction de la sécurité. En effet, face à la montée en puissance de la menace et des attaques terroristes, qui ne se produisent plus uniquement à l'extérieur du territorial national, les autorités publiques ont repensé l'organisation du renseignement, en passant d'une organisation "cloisonnée » à une "coopération entre les services [au moyen de] la collecte et $[\mathrm{du}]$ partage d'informations», ce qui permet aux services de renseignements " [d']analyser les risques, [d'anticiper] les menaces terroristes et d'assurer [une] stratégie de sécurité adaptée ${ }^{26} »$.

À ces dispositifs de concertation s'ajoutent des dispositifs dits opérationnels.

Ces dispositifs mis au service de la coproduction de la sécurité sont nombreux et ils abondent à l'échelle locale. Certains d'entre eux ont déjà été cités. Il importe toutefois de les rappeler. Il s'agit des CCPD créés en 1980; des CLSPD au niveau communal et des CISPD au

24. Ainsi désignée depuis le décret $\mathrm{n}^{\mathrm{0}}$ 2014-332 du 11 mars 2014 relatif à la Mission interministérielle de lutte contre la drogue et les conduites addictives. Placée auprès du Premier ministre, elle a pour rôle d'animer et de coordonner l'action du gouvernement en matière de lutte contre les drogues et les conduites addictives. Elle élabore à ce titre le plan gouvernemental et veille à sa mise en œuvre, http://www.drogues.gouv.fr/\#.

25. Virginie Gautron, art. cité.

26. Louis Gautier, «Renseignement et terrorisme», discours inaugural devant les sessions IHEDN-INHESJ, 9 décembre 2016, Paris, http://www.sgdsn.gouv.fr/discours/ renseignement-et-terrorisme. Les acteurs de cette coproduction du renseignement sont notamment la Direction générale de la sécurité extérieure; la Direction générale de la sécurité intérieure; la Direction du renseignement militaire; la Direction du renseignement de la sécurité et de la défense. 
niveau intercommunal. Ces institutions sont présidées par le maire ou le président de la structure intercommunale à fiscalité propre ${ }^{27}$. Leur composition est très variée et incarne l'esprit de partenariat, objectif qui leur est assigné. En effet, en sont membres : le maire, le président de la structure intercommunale à fiscalité propre, le préfet, le procureur de la République, le président du conseil départemental, les représentants des services de police de l'État dont la police et la gendarmerie nationales, les représentants d'associations, d'établissements, d'organismes intervenants dans le domaine de la prévention de la délinquance et de la sécurité ${ }^{28}$.

D’autres dispositifs opérationnels spécifiques ont également vu le jour. Nous pouvons citer notamment le $\mathrm{CDPD}^{29}$, présidé par le préfet, il intervient en matière d'aide aux victimes, de lutte contre la drogue, les dérives sectaires et les violences faites aux femmes et les groupes locaux de traitement de la délinquance créés par le parquet qui réunissent principalement le maire et les forces de police ou de gendarmerie. L'activation de ces dispositifs est limitée aux situations d'urgence, mais également dans l'espace (quartiers dits « sensibles») et dans le temps.

Au-delà de ces dispositifs, la dimension partenariale (contractualiste) de la sécurité apparaît au sein d'autres dispositifs opérationnels. Les plus connus sont les contrats locaux de sécurité $\left(\mathrm{CLS}^{30}\right)$. Institués par une

27. Les établissements publics de coopération intercommunale (EPCI) sont distingués "par leur degré d'intégration, reflété par leur régime fiscal: avec ou sans fiscalité propre». Ainsi, un EPCI est dit à fiscalité propre "[lorsqu'il] perçoi $[t]$ des ressources indépendantes des contributions communales", http://www.ladocumentationfrancaise.fr/dossiers/intercommunalite/cooperationintercommunale.shtm. Relèvent de cette catégorie d'EPCI: la communauté des communes, la communauté des communes à dotation globale de fonctionnement bonifiée, la communauté d'agglomération, la communauté urbaine, la métropole, les syndicats mixtes, les pôles métropolitains, les pôles d'équilibre territorial et rural, http://www.collectivites-locales.gouv.fr/differents-groupementsintercommunaux\#__RefHeading_56_587734144.

28. Depuis la loi $\mathrm{n}^{\mathrm{o}} 2013-403$ du 17 mai 2013 relative à l'élection des conseillers départementaux, des conseillers municipaux et des conseillers communautaires, l'assemblée dirigeante du département a changé de nom et devient conseil départemental.

29. Ce conseil est régi par le décret $\mathrm{n}^{0} 2006-665$ du 7 juin 2006 relatif à la réduction du nombre et à la simplification de la composition de diverses commissions administratives (art. D. 132-5 du Code de la sécurité intérieure).

30. Pour rappel, ces contrats ont remplacé les contrats d'action de prévention pour la sécurité dans la ville. Les diverses parties sont principalement le maire, le préfet et le procureur de la République, les recteurs ou leur représentant. 
circulaire du Premier ministre du 28 septembre $1997^{31}$, ils «marquent une évolution très importante dans les politiques publiques de sécurité [du fait qu'ils constituent] un nouveau dispositif de partenariat local» à l'originalité distinctive ${ }^{32}$. Cette originalité réside, en effet, dans la méthode précédant leur signature. Virginie Gautron et Nicolas Pannier indiquent respectivement que «ce dispositif contractuel doit être le point d'aboutissement d'une méthodologie rigoureuse» et que les contrats locaux de sécurité relèvent d'une "logique propre fondée sur une approche méthodologique rigoureuse ${ }^{33} »$. Ainsi, il apparaît que la conclusion des contrats locaux de sécurité exige un travail de fond en amont. Concrètement, ce travail méthodologique nécessite de la part des acteurs de la lutte contre l'insécurité de procéder à un état des lieux du niveau d'insécurité, des formes de celle-ci, des territoires concernés, des moyens - matériels et humains notamment - mobilisables, etc. : c'est le diagnostic local de sécurité (DLS). Ce DLS s'avère nécessaire en ce qu'il permet aux acteurs de prioriser leurs interventions, d'apporter des réponses rapides, efficaces et adaptées aux besoins des citoyens ${ }^{34}$.

Ces CLS traduiraient au mieux, l'idée d'une coproduction construite grâce aux échanges d'informations entre tous les acteurs (État, collectivités territoriales, associations, etc.) et au partage des moyens - humains et techniques. Mais, ils insistent davantage sur l'approche pragmatique et programmatique à laquelle doit s'attacher leur conclusion, et ceci, en vue d'atteindre un résultat. La reconduction de ces dispositifs en 2006 témoignerait de la positivité de leurs résultats ${ }^{35}$.

Pour finir, nous pouvons présenter d'autres dispositifs qui, à l'instar des CLS, traduisent également le recours à la technique contractuelle en

31. Circulaire relative à la mise en œuvre des contrats locaux de sécurité, JO 30 oct. 1997, $\mathrm{n}^{\mathrm{o}} 253$.

32. Nicolas Pannier, «Les contrats locaux de sécurité et la politique de la ville», RF aff. soc. 2001, p. 127-148.

33. Virginie Gautron, art. cité; Nicolas Pannier, art. cité.

34. La circulaire du Premier ministre du 28 octobre 1997 fixait ces objectifs : «Élaborés [les CLS] sous l'impulsion commune du préfet et du procureur de la République, avec les maires, ils permettront d'adapter la politique de sécurité aux réalités locales. Ils devront apporter à une situation d'insécurité particulière une réponse adaptée, globale et cohérente.»

35. Les CLS ont été reconduits par une circulaire interministérielle du 4 décembre 2006 sous la forme de CLS «nouvelle génération » pour prendre en compte l'évolution du contexte de la sécurité en France (qui nécessite une nouvelle politique de prévention de la délinquance dont la solution serait trouvée dans une meilleure coordination des actions sur les territoires), l'augmentation des violences aux personnes, la délinquance des mineurs et surtout la nécessité d'intégrer les CLS dans les contrats urbains de cohésion sociale. 
vue de la mise en œuvre des politiques de sécurité. Il s'agit des conventions de coordination. La mention expresse de ce type de dispositif permettant de rapprocher les forces de l'ordre figure à l'article 2 de la loi du 15 avril 1999 relative aux polices municipales ${ }^{36}$. Ces conventions peuvent être nationales ${ }^{37}$, locales ${ }^{38}$ ou être conclues entre les forces de police étatiques et les forces de police locales ${ }^{39}$. Il est à noter que la conclusion desdites conventions de coordination est rendue possible "dès lors qu'un service de police municipale comporte au moins cinq emplois d'agent de police municipale », mais cela est également possible «à la demande du maire, lorsqu'un service de police municipale compte moins de cinq emplois d'agent de police municipale ${ }^{40}$ ». À défaut de telles conventions, les interventions des policiers municipaux seraient limitées. La logique de coproduction par laquelle sont marquées lesdites conventions est relevée au niveau de la précision de «la nature et des lieux des interventions des agents de police municipale" ainsi que de la détermination des «modalités de coordination de ces interventions [d']avec celles de la police et de la gendarmerie nationales ${ }^{41} »$. Ce qui signifie qu'un échange d'informations et une certaine forme de mutualisation sont menés.

Les développements qui précèdent ont permis de mettre en évidence les principaux dispositifs - de concertation et opérationnels - qui matérialisent la politique de coproduction de la sécurité. Institués dans le but de coordonner les interventions des acteurs de la lutte contre l'insécurité et surtout de les rendre plus efficaces, certains dispositifs affichaient lors de leur institution un «impératif de résultats ». Il est dès lors souhaitable d'effectuer une recherche sur les résultats observables depuis leur mise en œuvre.

36. L. $\mathrm{n}^{\circ}$ 99-291, JO 16 avr. 1999, $\mathrm{n}^{\circ} 89$, p. 5607.

37. Lorsqu'elles ne concernent que les forces de police étatiques.

38. Lorsqu'elles concernent uniquement les polices municipales. Dans ce cas, la convention serait désignée comme "convention de coordination intercommunale " (D. $\mathrm{n}^{\mathrm{o}}$ 2012-2, 2 janv. 2012 relatif aux conventions types de coordination en matière de police municipale).

39. Cette dernière forme de convention de coordination semble être la plus fréquente.

40. Art. 2 de la loi du 15 avril 1999 relative aux polices municipales.

41. En l'absence de convention de coordination, les policiers municipaux ne pourront intervenir "qu'entre $6 \mathrm{~h}$ et $23 \mathrm{~h}$ à l'exception des gardes statiques des bâtiments communaux et de la surveillance des cérémonies, fêtes et réjouissances organisées par la commune» (art. 2 de la loi du 15 avril 1999). 


\subsection{Le bilan de la coproduction de la sécurité}

La coproduction de la sécurité est désormais une méthode ancrée dans les politiques de sécurité. La volonté de l'État de réorganiser sa gestion d'allocation de la sécurité en vue de lutter contre les maux qui portent atteinte à son ordre public et à l'intégrité physique ou matérielle des personnes s'est traduite par l'association de plusieurs acteurs aux profils divers. Les discours des politiques, notamment du président de la République, du ministre de l'Intérieur, des maires, des préfets, des présidents d'établissements publics de coopération intercommunale à fiscalité propre (EPCIFP) ne manquent pas de rappeler le changement d'aspect qui marque désormais la production de la sécurité ${ }^{42}$.

Ainsi, l'ancrage de la coproduction dans le système sécuritaire national nécessite de s'intéresser aux résultats qu'on peut y relever; autrement dit, il apparaît intéressant d'en dresser un bilan afin de savoir si les dispositifs créés ont atteint les objectifs qu'ils ambitionnaient et pour souligner d'éventuelles critiques. Toutefois, en dépit des critiques qui peuvent être formulées, on relève un certain nombre de résultats positifs.

Annonçons-le d'emblée, le bilan favorable que nous proposons de dresser autour de la coproduction de la sécurité ne sera pas exhaustif. Il ne fera certainement pas l'unanimité, car certaines études traitant de cette nouvelle approche d'allocation de la sécurité s'accordent plus à répertorier les failles de celle-ci qu'à contraster le bilan résultant de son effectivitét ${ }^{3}$. Les quelques lignes qui suivent entendent adopter la démarche inverse en s'intéressant tout d'abord aux résultats positifs de la coproduction de la sécurité avant de les contraster, car celle-ci reste perfectible. Dans cette perspective, deux niveaux d'appréciation de la positivité des résultats semblent pouvoir se dégager. Le premier niveau s'intéressera de façon générale à la mobilisation des acteurs que la coproduction de la sécurité a suscitée. Le second niveau portera sur l'état des lieux de l'insécurité dans ce contexte partenarial de la sécurité.

La lutte contre l'insécurité en général et contre les actes de violence et la délinquance en particulier exige de l'État et des collectivités territoriales (pour ce qui relève de leurs compétences) une réponse pénale aussi rapide qu'efficace. Mais ces deux principaux acteurs de la

42. Parfois prononcés dans des situations de crise.

43. Exception faite des travaux de Virginie Gautron qui, s'agissant notamment de l'appréciation des contrats locaux de sécurité, reconnaissent la positivité de leurs résultats tout en relevant quelques imperfections de la coproduction de la sécurité. Il se peut que d'autres travaux adoptent la même démarche. Mais nous tenons compte ici ceux disponibles au moment de la rédaction du présent article. 
sécurité ne sauraient, à eux seuls, remplir cette tâche. C'est la raison pour laquelle, la doctrine de la coproduction a favorisé l'intégration d'acteurs nouveaux et diversifiés à leurs côtés ${ }^{44}$.

La mobilisation de ces acteurs nouveaux constitue l'un des premiers résultats positifs de la doctrine de la coproduction. Cette dernière est parvenue à fédérer autour d'elle des intervenants dont la profession implique un mode de fonctionnement et de raisonnement souvent étranger à la vision centralisatrice de l'organisation de la sécurité. Désormais, les clivages, les inimitiés professionnelles, les dichotomies, etc., sont effacés au profit d'une réponse sociale de sécurité coproduite et nécessaire à la lutte contre une insécurité quasi permanente. Dans les faits, le recours à cette méthode de travail en commun semble avoir eu des effets positifs. Nous constatons, en effet, que «différents intervenants apprennent à s'écouter mutuellement, à dépasser des logiques d'imputation visant à attribuer aux autres la responsabilité des déficiences de l'action publique». Par ailleurs, nous notons la participation d'importants nouveaux acteurs: «commerçants, bailleurs [sociaux], pompiers, transporteurs, etc. ${ }^{45} "$.

Aux résultats issus de la mobilisation de nouveaux acteurs autour de la coproduction succèdent ceux émanant de la quantité des dispositifs tant de concertation qu'opérationnels qui ont permis de la concrétiser. En effet, plusieurs acteurs publics des politiques de sécurité ont ménagé l'institution de dispositifs de concertation, la conclusion, ainsi que la mise œuvre de dispositifs opérationnels. Sans procéder à une économie exhaustive du nombre de ces dispositifs, consacrons quelques lignes aux dispositifs opérationnels conclus ${ }^{46}$.

Concernant, par exemple, les CLS, deux ans après le colloque de Villepinte qui les institua, on en dénombrait 292, d'autres étaient en

44. Pascal Mbongo le souligne en avançant que la protection de l'ordre public (lato sensu) n'est pas (plus) l'apanage de l'État et de la commune, car interviennent actuellement dans le domaine de la protection de l'ordre public, «maires, préfets et procureurs dans une ingénierie institutionnelle et juridique que le législateur analyse $[\ldots]$ comme un alliage entre une logique partenariale (ou de co-production) et un pilotage par l'État» (Pascal Mbongo et Xavier Latour (dir.), Préfets, procureurs et maires. L'autorité publique au début du XXI siècle, Presses universitaires d'AixMarseille, 2011, p. 17.

45. Virginie Gautron, art. cité.

46. L'auteur se heurte à une difficulté d'accès aux sources, notamment une synthèse portant sur l'ensemble de dispositifs de concertation et opérationnels de coproduction de la sécurité. Les informations à sa portée sont nombreuses et diversifiées. Il a donc fait le choix de ne présenter que celles illustrant le mieux son propos. 
négociation ${ }^{47}$. Sous leur forme renouvelée (CLS dits de «nouvelle génération"), on en "dénombrait 687 [au 30 juin 2006] dont 195 [étaient] intercommunaux ${ }^{48} »$. Depuis lors, leur nombre a fortement augmenté, la majorité de villes en sont pourvues surtout celles dont le niveau d'insécurité est élevé ${ }^{49}$; les métropoles en sont également de plus en plus dotées ${ }^{50}$.

Pour leur part, les conventions de coordination entre les différentes forces de sécurité abondent également d'un territoire à l'autre, en fonction des besoins ${ }^{51}$.

En outre, nous pouvons aborder une dimension particulière que connaît actuellement la coproduction de la sécurité. Il s'agit de «la participation des personnes privées à la sécurité publique ${ }^{52} »$. Des agents privés de sécurité contribuent désormais à la protection des personnes et des biens dans les centres commerciaux, les aéroports, les gares, les salles de spectacles, devant les bâtiments ou encore lors de manifestations culturelles et sportives ${ }^{53}$. La contribution qu'apportent ces agents privés de sécurité est essentiellement d'ordre humain, matériel et technique.

47. Voir http://www.senat.fr/rap/r99-447-1/r99-447-143.html.

48. Virginie Gautron, art. cité.

49. Ce qui conduit généralement à leur insertion dans les zones dites de «sécurité prioritaire» (ZSP). À ce jour, il existe 8o ZSP : 24 en septembre 2012; 49 en novembre 2012; 16 en décembre 2013, http://www.interieur.gouv.fr/Archives/ Archives-des-actualites/2013-Actualites/ZSP/Combien-de-ZSP.

50. Paris, Lyon et Marseille disposent d'un contrat local de sécurité : contrat parisien de prévention et de sécurité 2015-2020, il a été renouvelé; la première échéance était prévue pour 2009-2013; Lyon fut l'une des premières villes en s'en doter en 1998, renouvelé en 2008 et actualisé aujourd'hui; Marseille n'est pas en reste, en dehors d'un CLS classique, la ville s'est dotée en 2009 d'un CLS applicable au domaine des transports publics. La métropolisation n'a pas arrêté la méthode de coproduction de la sécurité qu'expriment ces CLS, mais la confirme par l'actualisation des dispositifs. Nous pouvons donner l'exemple du récent CLS (2013-2018) de la métropole du Grand Nancy.

51. Charente-Maritime ( 7 en 2016 entre les forces de police étatiques et la police municipale); Marseille ( 1 en 2016 entre les forces de police étatiques et la police municipale); Le Loiret ( 1 en 2017 entre les forces de police étatiques et la police municipale); Nancy (le 20 janvier 2017 entre les forces de police étatiques et la police municipale).

52. Florence Nicoud, «La participation des personnes privées à la sécurité publique», RDP 2006, p. 1249; Xavier Latour, «La place du secteur privé dans la politique moderne de la sécurité », AJDA 2010, p. 657.

53. Ce partenariat s'est par exemple exprimé lors de l'Euro 2016 de football, au cours duquel «le besoin en agents de sécurité privée fut en moyenne de 925 par match» (Ziad Khoury, «La sécurité de l'Euro 2016 de football», Sécurité et Stratégie, $\mathrm{n}^{\circ}{ }_{23}$, 2016, p. 12). Frédéric Diaz, art. cité, p. 429-458. 
Enfin, nous pouvons apprécier les résultats de la coproduction de la sécurité en considérant la diminution ou la stabilisation de l'insécurité. Une telle appréciation produit des réponses variées tant le niveau d'insécurité, la forme d'insécurité combattue ainsi que les moyens dont disposent les acteurs de la sécurité pour protéger les citoyens sont euxmêmes aussi divers que variables. Peut-être faudrait-il raisonner de façon générale en évaluant tout simplement la politique de prévention de la délinquance, ce qui permettrait de voir comment les dispositifs de coproduction de la sécurité sont utilisés et quels en sont les résultats ${ }^{54}$. $\mathrm{Au}$ fond, c'est l'efficacité du fonctionnement des dispositifs partenariaux de sécurité qui est ici questionnée.

Au terme de cette étude portant sur l'appréciation des résultats de la coproduction de la sécurité, nous pouvons conclure que la mise en œuvre de cette méthode a généré des résultats positifs, mais une telle affirmation ne peut être que nuancée, car les dispositifs mis à son service ont également présenté quelques failles.

En effet, quelques failles ont pu être relevées dans le fonctionnement de la coproduction de la sécurité. Elles tiennent notamment au comportement des acteurs chargés de la matérialiser. En ce sens, on note au premier rang, "[l'ambivalence] du positionnement de l'État». Affichant sa volonté et sa détermination de promouvoir une nouvelle approche de la gestion de l'insécurité, son intervention manifeste en réalité, son intention " [de] restaurer, sinon [d']affermir, son autorité ». Cela apparaît notamment au niveau de sa capacité à définir et fixer les grandes orientations en matière de politiques de sécurité. Elle "préserve la dissymétrie des pouvoirs entre [lui] et ses partenaires »; ce qui semble s'éloigner de l'idée de coproduction. Une telle ambivalence de l'État desservirait la clarté, la cohésion et l'efficacité recherchées et impliquerait «la subordination des projets locaux aux exigences de l'État », ce dernier demeurant le principal architecte des politiques de sécurité.

Ensuite, le comportement des autres acteurs est lui aussi mis en cause et, particulièrement, l'abandon " [des] enceintes partenariales». En effet, «ils s'associent et se sollicitent ponctuellement, notamment dans des situations d'urgence» et «sur le long terme, leurs échanges restent

54. Pour avoir une idée sur la prévention de la délinquance (par exemple au niveau local et celle des jeunes), voir La Mission permanente d'évaluation de la politique de prévention de la délinquance, Gouvernance locale de la prévention de la délinquance, 2014 et La Mission permanente d'évaluation de la politique de prévention de la délinquance, Évaluation des dispositifs de prévention de la délinquance des jeunes, 2016. 
toutefois sporadiques ou s'essoufflent au fil du temps ${ }^{55} »$. Un rapport de la Mission permanente d'évaluation de la politique de prévention de la délinquance de septembre 2014 est venu confirmer cette désertion ${ }^{56}$. Par ailleurs, au sein des instances partenariales, des divergences d'opinions émergent entre les acteurs et notamment collectivités territoriales, ce qui ne favorise pas le développement « de rapports de complémentarité ». En définitive, c'est le cœur même de la coproduction de la sécurité qui est critiqué, autrement dit, les dispositifs créés pour la mettre en œuvre. Se pose alors la question de leur adéquation aux objectifs qui leur ont été assignés. Sur ce sujet, la première critique que lesdits dispositifs essuient est celle de leur instrumentalisation. En effet, il a été relevé que loin «d'expérimenter des projets novateurs définis collectivement, certaines administrations ont utilisé des crédits contractualisés pour financer leurs opérations de fonctionnement courant ${ }^{57}$ ». Ces critiques ont souvent concerné les premiers dispositifs opérationnels de la coproduction de la sécurité : les CLS ${ }^{58}$.

55. Virginie Gautron (art. cité) souligne que «les politiques pénales [constituent] une parfaite illustration de cette ambivalence [...]", (citant Sébastian Roché, op. cit., 1993) «parce que la légitimité de l'État dépend de sa capacité à rassurer et à protéger [...], la consécration du principe de coproduction coexiste avec la réaffirmation, bien qu'allusive, de son monopole pénal».

56. Fatiha Benatsou (dir.), Rapport sur la gouvernance locale de la prévention de la délinquance, 2014, 87 p. Les rapporteurs relèvent en effet que «dans les départements visités, la majorité de ces instances partenariales [CLSPD et CISPD] ne fonctionne pas, les maires ne réunissant pas leurs assemblées plénières" et «quand elle est réunie, l'assemblée plénière ne présente qu'un intérêt limité et qu'il s'agit souvent d'une "grande messe" au cours de laquelle aucune décision n'est prise».

57. Virginie Gautron, art. cité.

58. Hormis la question de leur adéquation aux objectifs poursuivis par la coproduction de la sécurité, c'est celle de leur nécessité qui est posée. En 2003, sur la base des conclusions de la première Mission interministérielle des CLS, certains auteurs notaient que "près de la moitié des contrats [avaient] été signés en dehors des départements les plus sensibles» (François Dieu et Bruno Domingo, "Partenariat et évaluation: le cas des contrats locaux de sécurité", Revue internationale de criminologie et de police technique, vol. LVI, $\mathrm{n}^{\mathrm{o}} 2$, 2003, p. 3-22, cités par Virginie Gautron, art. cité). En 2005, la mission dont la charge était de proposer des orientations pour la mise en place des CLS «nouvelle génération » avait confirmé ce constat en soulignant « tout à la fois le nombre élevé de CLS présents dans des petites et moyennes communes qui n'en avaient pas nécessairement besoin, leur caractère parfois trop ambitieux ainsi que le manque de suivi et d'animation de nombre d'entre eux" (Pierre Duffe et al., Propositions d'orientations pour la mise en place de contrats locaux de sécurité, Paris, Inspection générale de l'administration, 2005). Enfin, cette question de la nécessité a également été posée s'agissant des CLSPD (La Mission permanente d'évaluation de la politique de prévention de la délinquance, Gouvernance locale de la prévention de la délinquance, 2014, p. 6). 
En outre, l'efficacité de ces dispositifs a également été remise en cause du fait qu'en dépit de leur volonté affichée d'une meilleure coordination des « interventions des partenaires de la sécurité », maîtresmots lors de leur création, « [ces CLS ont peiné] à construire de véritables politiques de sécurité sur la base d'objectifs partagés ». Pour certains auteurs, «on a plus [eu] affaire à un bricolage local [notamment] dans la définition des programmes d'action qui relèvent davantage d'une compilation d'actions à caractère sectoriel et/ou communal que d'actions transversales, [et à un] bricolage dans le suivi et l'évaluation des dispositifs dont l'État est singulièrement absent, ou de manière insuffisante pour maîtriser le jeu ${ }^{59}$ ».

Cette critique conduit à en présenter une dernière qui semble formuler le regret d'un rendez-vous manqué entre l'État et les politiques publiques de la sécurité en général, et plus particulièrement, entre l'État et les politiques publiques locales de sécurité.

La réflexion menée sur l'appréciation de la coproduction de la sécurité, principalement de ces dispositifs, a permis de relever la part de positivité, de même que certains dysfonctionnements. Il en est résulté qu'en dépit des critiques qui peuvent être formulées à l'encontre de la coproduction de la sécurité et des améliorations souhaitées, il faut considérer ce bilan comme globalement positif. Par ailleurs, traduisant à certains égards un renouvellement de l'action publique de sécurité, la coproduction a encore de l'avenir devant elle, tant le besoin de sécurité se fait de plus en plus permanent. Elle connaîtra sans doute de futurs développements au

59. Tanguy Le Goff, «Les contrats locaux de sécurité à l'épreuve du terrain : réflexions sur l'action publique locale en matière de sécurité ", Politiques et management public, vol. $20, \mathrm{n}^{\mathrm{o}} 1$, 2002, p. 118. 


\author{
niveau nationa ${ }^{60}$ et extranational ${ }^{61}$. Elle s'améliorera éventuellement, \\ elle en a les potentialités ${ }^{62}$.
}

60. Au niveau national, nous pouvons affirmer que la coproduction de la sécurité bénéficie d'une garantie notamment législative: dans la loi n ${ }^{0}$ 95-73 d'orientation et de programmation relative à la sécurité du 21 janvier 1995 article $1^{\mathrm{er}}$, le législateur avait posé les jalons de cette forme de production de la sécurité en favorisant l'association de différents acteurs. Pour sa part, la loi n 2011-267 du 14 mars 2011 d'orientation et de programmation pour la performance de la sécurité intérieure n'a pas rompu avec les objectifs de nécessité d'une approche globale des politiques de sécurité, elle en fait un moyen essentiel pour la protection des citoyens (Annexe I, Assurer la sécurité partout et pour tous grâce à une approche globale de la politique de sécurité, 1 . Mobiliser tous les acteurs au service de la sécurité de nos concitoyens).

61. La montée en puissance des menaces et des attentats terroristes nécessite désormais des réponses pénales et des politiques de sécurité dépassant les seuls États-nations. Un élargissement extranational de la coproduction de la sécurité est donc possible (et même souhaitable) dans la mesure où la société actuelle est de plus en plus globalisée - ce qui favorise la mobilité des personnes et partant, de la criminalité -, les frontières nationales et limitrophes devraient faire l'objet d'une protection renforcée. Nous dénombrons déjà certains instruments qui concourent à la coopération internationale en matière de défense et de sécurité: notamment le Conseil de sécurité de l'ONU qui prend des mesures en matière d'opérations de maintien de la paix et de la sécurité internationales; l'OTAN qui intervient dans le domaine de la coopération politique, économique et militaire, dans le but de maintenir la paix et de gérer les conflits; l'Organisation pour la sécurité et la coopération en Europe dont les objectifs sont la stabilité et la sécurité des zones européennes. Au niveau de l'Union européenne, ce sont la politique étrangère et de sécurité commune et la politique européenne de sécurité et de défense qui matérialisent la politique étrangère de sécurité et de défense commune. Des collaborations plus étroites pourraient également se développer afin de renforcer les stratégies de sécurité déjà à l'œuvre. Au sein de l'Union européenne, par exemple, émerge en matière de sécurité une coopération public/privé. Elle procède de la volonté des États membres de construire un marché européen de la sécurité. De ce fait, ces derniers y consacrent notamment leurs finances (subventions) et instaurent des dialogues institutionnels. Voir Pierre Berthelet, «La coopération public/privé dans l'Europe de la sécurité: une politique industrielle en devenir", Sécurité et stratégie, $\mathrm{n}^{\circ}{ }_{23}, 2016$.

62. La coproduction de la sécurité pourrait se consolider davantage. Le contexte sécuritaire national et international marqué par la montée en puissance de l'insécurité et des vagues successives de menaces ainsi que d'attaques terroristes pourrait justifier cette consolidation; les acteurs de la sécurité intérieure étant résolument engagés à combattre conjointement ces formes d'insécurité. Cette consolidation pourrait se traduire par un rapprochement plus fort de tous les acteurs et par une coordination plus assurée de leurs interventions. Ne faudrait-il pas élaborer par exemple un schéma d'intervention (voir le schéma national d'intervention créé le 19 avril 2016 par le ministre de l'Intérieur pour lutter contre le terrorisme) qui, au-delà du seul rassemblement des acteurs de la sécurité, fixerait les rôles de chacun, prévoirait la répartition des moyens et préciserait les lieux d'intervention, notamment les lieux prioritaires. Ce schéma aurait l'avantage et le mérite d'incarner une véritable stratégie, au sein de laquelle l'État assurerait le pilotage tout en le décentralisant pour 
Les développements précédents se sont intéressés à l'avènement de la coproduction de la sécurité. Nouvelle doctrine de production de la sécurité, elle s'est essentiellement construite au sein des politiques publiques de lutte contre l'insécurité. Pour la matérialiser, plusieurs dispositifs, de concertation ou opérationnels, ont été créés. Les résultats qu'ils ont pu permettre d'obtenir en termes de diminution de l'insécurité ont également fait l'objet d'une analyse. En outre, certains de ses acteurs ont été cités sans toutefois que leur présentation ait fait l'objet d'un approfondissement. C'est à ces acteurs que les lignes qui suivent s'intéresseront.

\section{Les acteurs de la coproduction de la sécurité}

Divers acteurs participent de nos jours à la production de la sécurité des personnes et des biens (2.1) ; leur association dans ce domaine traduit une certaine quête d'efficacité (2.2).

\subsection{La participation de divers acteurs à la production de la sécurité}

Si en principe, "la nation contribue à sa propre défense [et] à la disponibilité de sa force», il «[apparaît que] l'union de tous [pour la

une meilleure productivité. Bien des éléments permettent d'espérer que la coproduction public/public de la sécurité a encore de beaux jours devant elle et plusieurs domaines à développer, de même que la coproduction public/privé. Concernant cette dernière tout semble conduire à affirmer qu'elle va s'affermir progressivement eu égard « [au] manque de moyens financiers et d'effectifs [qui conduit] la puissance publique à déléguer un nombre croissant de missions [de sécurité] à des personnes privées » (Perrine Dumas, «Les personnes privées et les activités de souveraineté: réflexions sur l'externalisation des activités de sécurité ", Annales de droit, $\mathrm{n}^{\circ}$ 6, 2012, p. 67) produisant de facto une externalisation des activités de souveraineté (JeanDavid Dreyfus, «L'externalisation, éléments de droit public », AJDA 2002, p. 1214; Philippe Cossalter, «Le droit de l'externalisation des activités publiques dans les principaux systèmes européens", séminaire mensuel sur le droit administratif comparé, européen et global, 23 mars 2007, http://www.sciencespo.fr/chaire-madp/ sites/sciencespo.fr.chaire-madp/files/cossalter.pdf). Elle va d'autant plus s'affermir que le secteur de la sécurité privée ne cesse de gagner en confiance et en professionnalisation. Cela se vérifie pour ce qui touche à la sécurité ordinaire, cela s'est particulièrement confirmé lors de l'Euro 2016.Un tel «succès [devrait] désormais permettre d'aller vers une véritable doctrine d'emploi de la sécurité privée dans l'architecture globale de la sécurité intérieure » (Jean-Paul Célet, «L'EURO 2016 : les fondements d'une doctrine d'emploi de la sécurité privée dans l'architecture de la sécurité intérieure ", Sécurité et stratégie, $\mathrm{n}^{\circ}{ }_{23}$, 2016, p. 26. Pour rappel, Jean-Paul Célet (préfet) est l'actuel directeur du Conseil national des activités privées de sécurité. 
produire] fait la force ${ }^{63}{ }^{\prime}$. Cette assertion invite à opérer l'interprétation suivante: d'une part, le rôle de l'État comme premier garant de la sécurité des personnes et des biens est affirmé; d'autre part, la production de la sécurité n'émanerait plus uniquement de l'État, à ses côtés, il faut désormais compter l'intervention d'autres acteurs. Cette mutation de la production et de l'organisation de la sécurité constituerait une adaptation de l'État au contexte social marqué par des formes diverses d'insécurité et lutter contre elles nécessite de faire appel autant aux moyens de la police judiciaire, de la police administrative, des criminologues qu'à ceux des associations, des affaires sociales, des éducateurs, des psychologues, etc.; elle prendrait également en compte les difficultés (matérielles, budgétaires, etc.) auxquelles sont parfois confrontés l'État et les collectivités dans leur gestion des charges liées à la sécurité.

Ainsi, l'architecture des acteurs de la production de la sécurité connaît une reconfiguration, conformément à la doctrine de coproduction. Elle peut se décliner de la façon suivante: tout d'abord les acteurs principaux (l'État, central et déconcentré) et les collectivités territoriales; ensuite, les acteurs additionnels (les associations, les bailleurs sociaux, les éducateurs, les criminologues, la sécurité privée $\left.{ }^{64} . ..\right)$.

$\mathrm{Au}$ sein du premier groupe d'acteurs, l'État se présente comme le producteur naturel de la sécurité des personnes et des biens. En effet, selon des conceptions fort anciennes (Hobbes, Machiavel, Delamare, Rousseau, Max Weber, etc.), l'usage de la force relève de son apanage. Il définit à ce titre les grandes orientations des politiques de sécurité et punit les personnes qui, par leur comportement, troublent l'ordre public, commettent une infraction, etc.

Apparaissent ensuite les collectivités territoriales : notamment la commune (le maire) et le département ${ }^{65}$ (le préfet). À ces deux principales autorités de police locale, peut être ajouté le président d'EPCIFP dont les pouvoirs de police n'ont cessé de s'élargir ces dernières années ${ }^{66}$. Enfin,

63. Christian Vigouroux, Du juste exercice de la force, op. cit., p. 274 et 272.

64. Concernant cet ensemble d'acteurs, voir André-Michel Ventre, «Les polices en France», Pouvoirs, $\mathrm{n}^{\mathrm{o}}$ 102, 2002, p. 31-42.

65. Art. L. 2212-2 du CGCT définissant la police municipale et art. L. 2215-1 du CGCT.

66. L. $\mathrm{n}^{0}$ 2004-809, 13 août 2004 relative aux libertés et responsabilités locales. Elle avait, outre le transfert du personnel des collectivités territoriales aux intercommunalités, initié le transfert (facultatif) des pouvoirs de police spéciale des maires aux présidents d'EPCIFP. Ce transfert est devenu obligatoire à l'issue de la loi n ${ }^{\circ}$ 2010-1563 du 16 décembre 2010 de réforme des collectivités territoriales. Les récentes lois $\mathrm{n}^{\mathrm{o}} 2014-58$ du 27 janvier 2014 de modernisation de l'action publique territoriale et d'affirmation des métropoles et $\mathrm{n}^{\mathrm{o}} 2015-991$ du 7 août 2015 portant nouvelle organisation territoriale de la République ont confirmé ce transfert. 
prennent également une part active en matière de sécurité, des acteurs aux profils diversifiés qui constituent pour l'État et les collectivités territoriales un important soutien, notamment pour les politiques de lutte contre la délinquance. Parmi eux, c'est le rôle des agents privés de sécurité qui a davantage retenu l'attention ces dernières années tant leur intervention en matière de sécurité devient fréquente et tant leur secteur d'activités évolue progressivement vers la constitution d'un véritable marché privé de la sécurité dont le fonctionnement nécessiterait une régulation ${ }^{67}$.

Ces acteurs mobilisent chacun des moyens humains, matériels et économiques pour coproduire la sécurité. S'il est incontestable que pour aboutir à cette "nouvelle culture de la sécurité », leur association doit être davantage facilitée, elle ne devrait toutefois pas conduire à leur uniformisation, mais plutôt à leur complémentarité ${ }^{68}$. De là découlent quelques aspects de leur articulation: l'État est le décideur central des politiques de sécurité, il en fixe les grandes orientations et les modalités d'exécution. Concernant ses rapports avec les entreprises privées de sécurité, c'est lui qui organise, par exemple, leurs interventions afin de distinguer les tâches qu'elles peuvent prendre en charge de celles qu'il entend réserver à ses propres agents ${ }^{69}$; qu'à cela ne tienne, ces entreprises contribuent activement à la protection des personnes et des biens.

67. Leur investissement dans la gestion des récents attentats terroristes et dans la sécurité de l'Euro 2016 permet de le vérifier (en ce qui concerne la sécurité de l'Euro 2016 par les agents privés de sécurité, 13000 agents privés de sécurité ont été mobilisés, voir Euro 2016, Civique, hors-série n ${ }^{\circ}$ 3, p. 26).

68. Christian Vigouroux, Du juste exercice de la force, op. cit., p. 280 et 273.

69. Nous rappelons sur ce point, les jurisprudences de principe qui interdisent la délégation aux personnes privées de l'exercice des pouvoirs de police: $\mathrm{CE}$, ass., 17 juin 1932, req. $\mathrm{n}^{\mathrm{O}}{ }_{12045}$, Ville de Castelnaudary: Rec. Lebon, p. 595 ; CE, sect., $1^{\text {er }}$ avr. 1994, req. $n^{\circ} 144152 / 144241$, Commune de Menton ; CE, sect., 29 déc. 1997 , req. $\mathrm{n}^{\circ}$ 170606, Commune d'Ostricourt: Rec. Lebon; TA Montpellier, 5 juil. 2016, $\mathrm{n}^{\mathrm{o}}$ 1506696, "Garde bitérroise »; Cons. const., déc. n 2011-625 DC, 10 mars 2011 relative à la loi d'orientation et de programmation pour la performance de la sécurité intérieure. Frédéric Ocqueteau souligne que l'État agit dans ce cas comme un «régulateur, c'est lui qui est capable d'autoriser et de réguler des systèmes de gouvernance privés [de sécurité] » (Polices entre État et marché, Paris, Presses de Sciences Po, 2004, p. 172-173). Nous rappelons également la loi $\mathrm{n}^{0} 83-629 \mathrm{du}$ 12 juillet 1983 réglementant les activités privées de sécurité; l'institution en 2011, du Conseil national des activités privées de sécurité chargé de contrôler ce secteur d'activités ainsi que la récente loi ${ }^{\circ}$ 2017-258 du 28 février 2017 relative à la sécurité publique qui encadre leur possibilité d'usage des armes. 
S'agissant enfin de ses rapports avec les collectivités territoriales, ils ne se signalent pas par la concurrence, mais plutôt par un véritable partenariat. En effet, les élus locaux constituent des partenaires privilégiés de l'État territorial, tant ce sont ces derniers qui mettent en œuvre les politiques publiques de sécurité qu'il définit, assurent et coordonnent celles qui ont vocation à s'appliquer sur l'ensemble du territoire national ${ }^{70}$. À ce titre, l'État les dote de moyens nécessaires et leur attribue parfois une mission de pilotage de ses politiques. En tout état de cause, l'État a intérêt à s'associer aux collectivités territoriales, acteurs incontournables de la mise en œuvre et de l'exécution de ses politiques de sécurité; celles-ci ont tout autant besoin des moyens de l'État pour assurer la sécurité de leurs territoires.

Dans le cadre de la coproduction de la sécurité, ces acteurs partagent leur expérience et leurs pratiques de fonctionnement ou encore leur stratégie d'action. La politique de prévention de la délinquance est l'un des principaux domaines où s'affirme cette coproduction ${ }^{71}$. En effet, elle « associe [notamment] l'État, ses autorités administratives et judiciaires, les services de l'éducation, de la santé, de la jeunesse et des sports, les collectivités territoriales, les associations, et aussi les acteurs du logement de la santé ${ }^{72}$ ». Toutefois, l'introduction de la coproduction dans la gestion de la sécurité a rouvert le débat autour du monopole dont l'État est censé être attributaire en matière de sécurité. La question est de savoir comment interpréter le partage de son pouvoir d'action et de décision en matière de police avec d'autres acteurs ${ }^{73}$. Sur ce point, Sébastian Roché avance que ce partage conduit à la réalisation « [d']une démonopolisation de l'exercice de la sécurité ${ }^{74} »$ au détriment de l'État;

70. Voir la stratégie nationale de prévention de la délinquance 2013-2017 fixée par l'État central, au sein de laquelle la place des maires et des préfets est activement consacrée.

71. Nous signalons qu'au sens de la loi $\mathrm{n}^{0} 2007-297$ du 5 mars 2007 relative à la prévention de la délinquance, le maire est le principal acteur de la politique de prévention de la délinquance.

72. La mise en œuvre de cette coproduction conduit les différents acteurs de s'organiser " en réseau, avec l'institutionnalisation de groupes, de réunion, des diagnostics et des objectifs partagés, des actions communes, des financements croisés, etc.» (Francis Lamy, «La production de la sécurité publique », Archives de philosophie de droit, t. 58,2015, p. 23).

73. Cette question s'est d'abord posée en raison de l'intervention des autorités locales en matière de sécurité, puis de celle des entreprises privées de sécurité. Elle se pose enfin également eu égard aux politiques européennes de sécurité qui impactent l'État.

74. Sébastian Roché, «Vers la démonopolisation des fonctions régaliennes : contractualisation, territorialisation et européanisation de la sécurité intérieure », RFSP, vol. 54, $\mathrm{n}^{\mathrm{o}} 1,2004$, p. 44. 
pour sa part, Jacques Chevallier se demande si « la police [est] encore une activité régalienne ${ }^{75} »$.

Pour répondre aux questions suscitées par ce débat, il faut considérer que l'élargissement de la production de la sécurité à d'autres acteurs est nécessaire, eu égard à la diversification des causes de l'insécurité et des moyens mobilisés pour y remédier; le rôle de l'État ne saurait être banalisé, son monopole en matière de sécurité ne subit qu'une dilution.

$\mathrm{Au}$ regard de l'ensemble des développements précédents, l'institution de la force obéit, d'une part à un besoin de défense de l'État contre d'éventuelles attaques ou menaces qui compromettraient son intégrité territoriale et, d'autre part, elle répond à celui de protection des personnes. Ainsi, sa mise en œuvre doit être utile à la satisfaction de ce besoin, elle doit encore être nécessaire pour le réaliser et surtout être adaptée à la menace ou au comportement répréhensible. Au-delà de l'énoncé de ces quelques finalités, la production de la sécurité n'attacherait-elle pas également du prix à l'efficacité?

\subsection{Le recours à la coproduction, quête d'efficacité des politiques de sécurité?}

À y regarder de plus près, le recours à la coproduction de la sécurité révèle le souci pour ses acteurs de rendre meilleure la production de la sécurité. Autrement dit, le partage de leurs moyens humains, matériels et économiques ainsi que de leur expérience et leurs pratiques de fonctionnement doit les conduire à une action utile, non seulement pour faire reculer l'insécurité, mais également pour mieux protéger le citoyen. Cette entreprise ne conduit-elle pas à assigner à la production de la sécurité des personnes et des biens des objectifs d'efficacité ou d'efficience voire de qualité?

$\mathrm{Au}$ vu de la profonde métamorphose dont ne cesse de faire l'objet l'administration de la sécurité, la réponse que nous pouvons apporter à cette question semble positive. En effet, nous constatons depuis quelques années l'introduction des pratiques de management dans les services de police ce qui constitue "une révolution", car ces pratiques de management «impliquent une nouvelle accountability [c'est-à-dire une sorte de] reddition des comptes» imposée à la production de la

75. Jacques Chevallier, «La police est-elle encore une activité régalienne? », Archives de politique criminelle, vol. $1, \mathrm{n}^{\mathrm{o}} 33,2011$, p. 13-27; « La police est-elle encore une activité régalienne? », dans Charles Vautrot-Schwarz (dir.), La police administrative, Paris, PUF, 2014. 
sécurité $^{76}$. En dehors du domaine de la sécurité, plusieurs pans de l'action administrative semblent désormais marqués par cette recherche d'efficacité ${ }^{77}$.

De façon générale, l'introduction d'objectifs d'efficacité et d'efficience dans la gestion de la sécurité conduit les pouvoirs publics à recourir à divers instruments comme les statistiques pour mesurer l'état de la criminalité et de la délinquance et pour adapter l'outil répressif et de protection aux «données » résultant de ces statistiques ${ }^{78}$; dans le même ordre d'idées, ces instruments peuvent servir à vérifier l'action policière sur le terrain tant sa présence doit rassurer, dissuader, contraindre et punir en cas de nécessité ${ }^{79}$. Par ailleurs, l'augmentation du budget des domaines régaliens pourrait également illustrer ce nouvel aspect de la gestion de la sécurité.

Pour le sujet qui nous intéresse, cette quête d'efficacité se traduit par des processus de programmation et de coordination des politiques de sécurité afin d'éviter des interventions éparses qui nuiraient à la lisibilité de ces politiques et surtout à leur mise en œuvre qui doit être faite dans des conditions adéquates. Nous relevons également des processus de territorialisation dont l'objectif est « de mieux prendre en compte de la variabilité et la multiplicité des situations locales» afin

76. Pour une idée de définition du terme management, nous présentons celle proposée par le Centre national des ressources textuelles et lexicales: le management est un «ensemble des méthodes d'organisation efficace (définition et partage des responsabilités) et de gestion rationnelle (en fonction d'objectifs ou de programmes fixés) employées dans la direction d'une affaire, d'une entreprise». Voir également Frédéric Ocqueteau, op. cit., p. 173-181. L'auteur souligne que cela renvoie à "une obligation de rendre compte ou d'offrir des explications a posteriori des conduites des agents par rapport aux réformes engagées». Le recours aux notions de "produit», "client» et de "satisfaction du client» traduirait ces normes de management (public).

77. En ce sens, la loi organique $\mathrm{n}^{\mathrm{0}} 2001-692 \mathrm{du} 1^{\mathrm{er}}$ août 2001 relative aux lois de finances a été un des vecteurs de la diffusion des indicateurs de la performance dans l'administration.

78. Nous indiquons sur ce point, l'intervention dans ce domaine, de l'Observatoire national de la délinquance et de la réponse faisant partie de l'Institut national des hautes études de la sécurité et de la justice.

79. On a parlé à un moment donné de «la culture du chiffre». L'action de Nicolas Sarkozy, à l'époque ministre de l'Intérieur, en avait été particulièrement marquée. Jean-Hugues Matelly et Christian Mouhanna, Police: des chiffres et des doutes. Regard critique sur les statistiques de la délinquance, Paris, Michalon, 2007, 269 p.; Sébastian Roché, "Culture du chiffre et efficacité de la police», La Tribune, 23 avril 2017. 
d'apporter aux territoires une réponse adaptée à leurs besoins ${ }^{80}$. Ainsi, conformément à cet esprit managérial de la sécurité, la coproduction a fait l'objet de plusieurs évaluations, notamment en matière de politiques de prévention de la délinquance ${ }^{81}$. Ces évaluations sont le signe de l'intérêt que l'État et les autres acteurs de la sécurité accordent au résultat de leur action.

Pour finir, la quête par les acteurs de la coproduction de l'efficacité des politiques de sécurité peut se justifier principalement en raison d'une demande sociale de sécurité elle-même croissante, voire pressante. Ainsi, il s'agit pour l'État, les collectivités territoriales ainsi que leurs partenaires de faire cesser le trouble social sous toutes ses formes et d'assurer par tous les moyens la protection du citoyen et celle de ses biens, et ce, de façon parfois urgente.

L'intégration des objectifs d'efficacité, d'efficience, voire de qualité dans les politiques publiques de sécurité s'avère nécessaire afin de mesurer, notamment, le degré d'effectivité des actions programmées pour répondre de façon adéquate au besoin multiforme de sécurité exprimé par le citoyen ou de faire reculer la criminalité.

La protection des personnes et des biens constitue de nos jours une préoccupation sociale partagée. En effet, si la sécurité relève d'abord de la responsabilité de l'État et des collectivités territoriales, sa production ne saurait plus relever de leur droit exclusif. Elle «[est] un bien commun : elle mérite la solidarité ${ }^{82} »$. La coproduction à laquelle nous nous sommes intéressé rendrait compte de cette solidarité, qui permet l'association de divers acteurs dans la lutte contre l'insécurité protéiforme; elle se vérifie particulièrement dans les politiques de prévention de la délinquance au sein desquelles interviennent l'État, les collectivités territoriales, les parquets, les associations, les bailleurs sociaux, les entreprises privées de sécurité, etc.

80. Olivier David, «La territorialisation des politiques publiques et cohésion nationale: un mariage complexe », dans Territoires en action et dans l'action, Presses universitaires de Rennes, 2008, p. 1.

81. Voir http://www.interieur.gouv.fr/SG-CIPDR/Ressources2/Mission-permanented-evaluation/L-evaluation-pour-mesurer-l-impact-des-politiques-de-preventionde-la-delinquance. Le guide de l'évaluation des politiques locales de prévention de la délinquance (2011) indiquait d'emblée que l'évaluation des politiques de prévention de la délinquance avait pour principal but de «mesurer l'efficacité des actions menées".

82. Éric Delbecque, «Une perspective historique: de l'État Providence à la société de défiance», dans Pierre Brajeux, Éric Delbecque et Michel Mathieu (dir.), Sécurité privée, enjeu public, Paris, Armand Colin, 2013, p. 39. 
Cette coproduction signalerait une mutation de l'État dans ses modes de fonctionnement en général et ceux de gestion de la sécurité en particulier. D'une part, le contexte social national et international l'exige, d'autre part, les difficultés humaines, financières et matérielles auxquelles il est parfois confronté l'y contraignent. De ce fait, c'est la conception même de la sécurité qui se reconfigure, mais sans que cette reconfiguration conduise à une relégation de la place de l'État régalien dans sa fonction de maintien de l'ordre et de garantie des libertés des citoyens.

Doctorant en droit public, chargé d'enseignement Université de Lorraine (IRENEE-EA 703) 